\title{
Stability of a Paddy Ecosystem with Time Delay
}

\author{
Yi Wang, Xiaoli Zhou, Zhaohua Wu and Tiejun Zhou* \\ College of Science, Hunan Agricultural University, Changsha, Hunan 410128, China \\ ${ }^{*}$ Corresponding author
}

\begin{abstract}
A delay differential equation model of paddy ecosystem was put forward to reveal the interaction among rice, weeds and inorganic fertilizer on the system. The results show that, the system exists a rice and weed extinction equilibrium, and it also exists a rice extinction or weed extinction equilibrium. Their stable and unstable conditions are obtained. Moreover, Hopf bifurcations occur at the rice extinction or weed extinction equilibrium as the delay crosses some critical values. According to the conditions, some measures to increase rice yield were recommended.
\end{abstract}

Keywords-paddy ecosystem; delay; equilibrium; stability; Hopf bifurcation

\section{INTRODUCTION}

As is well know, there are a lot of components in the paddy ecosystem, such as rice, weed, insect, microorganism, inorganic fertilizer, light intensity, moisture. The interaction of the components is a complex nonlinear relationship, and it also is a process of their survival, evolution and adaptation in the environment. At present, the research on paddy ecosystem is mainly concentrated in the field experiment and data analysis [1-3].

In order to describe quantitatively the change of material and energy in the paddy ecosystem, we need construct a dynamics model to reflect their conversion characteristics. Using this mathematical model, we can predict the yield of rice, we also can analyze the evolution law of soil fertility. But there are still not the matured mathematical models such as those in the forest and marine ecosystems [4-7].

The main purpose of this paper is to establish a differential equation model for the interaction among the main components of a paddy ecosystem, and to analyze the existence and stability of the system's equailibria.

\section{The Modeling of A PAdDy ECOSYSTEM}

We only consider three main components of paddy ecosystem: rice, weed and inorganic fertilizer. The growth of rice and weeds are affected by soil fertility, light and other factors. There is natural death for the rice and weed. The inorganic fertilizer in soil partly comes from fertilization and partly comes from organic fertilizer such as decaying leaves of rice and weed, which can are transformed to inorganic fertilizer after some time by microbial. Natural loss also reduces the content of inorganic fertilizers in soil.

According to the interaction relationship, a dynamic model of composite farming paddy ecosystem is established as follows,

$$
\left\{\begin{aligned}
\dot{r}= & c_{1} s_{1} u(t) r(t)-d_{1} r(t), \\
\dot{p}= & c_{2} s_{2} u(t) p(t)-d_{2} p(t), \\
\dot{u}= & b+d_{1} r(t-\tau)+d_{2} p(t-\tau) \\
& -s_{1} u(t) r(t)-s_{2} u(t) p(t)-d_{3} u(t) .
\end{aligned}\right.
$$

where $r(t)$ denotes the rice biomass per unit area at time $t, p(t)$ denotes the weed biomass per unit area at time $t$, and $u(t)$ denotes the inorganic fertilizer content per unit area at time $t$. In system (1), we assume that the uptake of inorganic fertilizer by rice and weed follows the mass action law.

On the right hand side of the first two equations in system (1), the $c_{1} S_{1} u r$ and $c_{2} S_{2} u p$ represent rice and weed growth rate, respectively, and the $d_{1} r$ and $d_{2} p$ are the mortality of rice and weed. The rice and weed growth rates are affected by light intensity $I$ and inorganic fertilizer $u$. The parameters $s_{i}$ are a light effects $(i=1,2)$.

On the right hand side of the third equation in system (1), the first term represents artificial fertilizer rate. The second and the third terms are inorganic fertilizers transformed from dead rice and weeds at $t-\tau$ time. The fourth and the fifth terms are the consumption of inorganic fertilizer by the growth of rice and weed. The last item indicates the loss of inorganic fertilizer in paddy field.

According to the actual requirements, the parameters in system (1) are non negative, and satisfy the following conditions $0<c_{i} \leq 1, b \geq 0$ and $d_{i}>0$.

For the sake of convenience, we introduce the following notations,

$$
\theta_{1}=\frac{d_{1}}{c_{1} s_{1}}, \theta_{2}=\frac{d_{2}}{c_{2} s_{2}}
$$

where $\theta_{1}$ is called the relative mortality of rice, $\theta_{2}$ is called the relative mortality of weed.

\section{THE EXISTENCE AND STABILITY OF EQUILIBRIA}

An equilibrium $\left(r^{*}, p^{*}, u^{*}\right)$ of system (1) satisfies the following equations, 


$$
\begin{aligned}
& F_{1}\left(r^{*}, p^{*}, u^{*}\right)=c_{1} s_{1} u^{*} r^{*}-d_{1} r^{*}=0, \\
& F_{2}\left(r^{*}, p^{*}, u^{*}\right)=c_{2} s_{2} u^{*} p^{*}-d_{2} p^{*}=0, \\
& F_{3}\left(r^{*}, p^{*}, u^{*}\right)=b+d_{1} r^{*}+d_{2} p^{*}-s_{1} u^{*} r^{*}-s_{2} u^{*} p^{*}-d_{3} u^{*}=0 .
\end{aligned}
$$

We make a coordinate transformation $x=r-r^{*}, y=p-p^{*}, z=u-$ $u^{*}$, then system (1) can be converted to

$$
\left\{\begin{aligned}
\dot{x}(t)= & c_{1} s_{1}\left(u^{*}-\theta_{1}\right) x(t)+c_{1} s_{1} r^{*} z(t)+c_{1} s_{1} x(t) z(t), \\
\dot{y}(t)= & c_{2} s_{2}\left(u^{*}-\theta_{2}\right) y(t)+c_{2} s_{2} p^{*} z(t)+c_{2} s_{2} y(t) z(t), \\
\dot{z}(t)= & d_{1} x(t-\tau)-s_{1} u^{*} x(t)+d_{2} y(t-\tau)-s_{2} u^{*} y(t) \\
& -\left(s_{1} r^{*}+s_{2} p^{*}+d_{3}\right) z(t)-s_{1} x(t) z(t)-s_{2} y(t) z(t) .
\end{aligned}\right.
$$

is

So the characteristic equation of the linearized system of (1)

$$
\left|\begin{array}{rrr}
\lambda-c_{1} s_{1}\left(u^{*}-\theta_{1}\right) & 0 & -c_{1} s_{1} r^{*} \\
0 & \lambda-c_{2} s_{2}\left(u^{*}-\theta_{2}\right) & -c_{2} s_{2} p^{*} \\
s_{1} u^{*}-d_{1} e^{-\lambda \tau} & s_{2} u^{*}-d_{2} e^{-\lambda \tau} & \lambda+s_{1} r^{*}+s_{2} p^{*}+d_{3}
\end{array}\right|=0 .
$$

From $F_{1}\left(r^{*}, p^{*}, u^{*}\right)=0$, we have $r^{*}\left(c_{1} s_{1} u^{*}-d_{1}\right)=0$. Therefore, we obtain

$$
r^{*}=0, \text { or } u^{*}=\theta_{1} \text {. }
$$

From $F_{2}\left(r^{*}, p^{*}, u^{*}\right)=0$, we have $p^{*}\left(c_{2} s_{2} u^{*}-d_{2}\right)=0$. Therefore, we know

$$
p^{*}=0, \text { or } u^{*}=\theta_{2} \text {. }
$$

Thus we can calculate the equilibria of system (1) in three cases as follows.

Case (I), $r^{*}=0$ and $p^{*}=0$. At this case, we have the following conclusion of the existence and stability of equilibrium.

Theorem 1. There exists a paddy and weed extinction equilibrium of system (1), given by

$$
\left(r_{1}^{*}, p_{1}^{*}, u_{1}^{*}\right)=\left(0,0, b / d_{3}\right)
$$

If $b / d_{3}<\min \left\{\theta_{1}, \theta_{2}\right\}$, the equilibrium $\left(r_{1}{ }^{*}, p_{1}{ }^{*}, u_{1}{ }^{*}\right)$ is locally asymptotically stable. Otherwise, If $b / d_{3}>\min \left\{\theta_{1}, \theta_{2}\right\}$, the equilibrium $\left(r_{1}{ }^{*}, p_{1}{ }^{*}, u_{1}{ }^{*}\right)$ is unstable.

Proof. Substituting $r^{*}=0$ and $p^{*}=0$ into $F_{3}\left(r^{*}, p^{*}, u^{*}\right)=0$, we have $u^{*}=b / d_{3}$. Therefore, there exists a paddy and weed extinction equilibrium $\left(r_{1}{ }^{*}, p_{1}{ }^{*}, u_{1}{ }^{*}\right)=\left(0,0, b / d_{3}\right)$ of system (1). At the equilibrium $\left(r_{1}{ }^{*}, p_{1}{ }^{*}, u_{1}{ }^{*}\right)$, the characteristic equation of the linearized system is as follows from (4)

$$
\left|\begin{array}{rrr}
\lambda-c_{1} s_{1}\left(u_{1}^{*}-\theta_{1}\right) & 0 & 0 \\
0 & \lambda-c_{2} s_{2}\left(u_{1}^{*}-\theta_{2}\right) & 0 \\
s_{1} u_{1}^{*}-d_{1} e^{-\lambda \tau} & s_{2} u_{1}^{*}-d_{2} e^{-\lambda \tau} & \lambda+d_{3}
\end{array}\right|=0 .
$$

It has three eigenvalues $\lambda_{1}=-d_{3}<0, \lambda_{2}=c_{1} s_{1}\left(b / d_{3}-\theta_{1}\right)$, and $\lambda_{3}=c_{2} s_{2}\left(b / d_{3}-\theta_{2}\right)$.

If $b / d_{3}<\min \left\{\theta_{1}, \theta_{2}\right\}$, the eigenvalues $\lambda_{2}<0$ and $\lambda_{3}<0$. Therefore, the equilibrium $\left(r_{1}{ }^{*}, p_{1}{ }^{*}, u_{1}{ }^{*}\right)$ of system (1) is locally asymptotically stable.

If $b / d_{3}>\min \left\{\theta_{1}, \theta_{2}\right\}$, at least one of the eigenvalues $\lambda_{2}$ and $\lambda_{3}$ is positive. Therefore, the equilibrium $\left(r_{1}{ }^{*}, p_{1}{ }^{*}, u_{1}{ }^{*}\right)$ is unstable under this condition.

Case (II), $p^{*}=0$ and $u^{*}=\theta_{1}$. From $F_{3}\left(r^{*}, p^{*}, u^{*}\right)=0$, we have

$$
r^{*}=\frac{b-d_{3} \theta_{1}}{s_{1} \theta_{1}\left(1-c_{1}\right)}
$$

Therefore, we obtain the conclusion of the existences and stability of equilibrium at the case (II).

Theorem 2. If $b / d_{3}>\theta_{1}$, then system (1) has a equilibrium $\left(r_{2}{ }^{*}, p_{2}{ }^{*}, u_{2}{ }^{*}\right)$, where

$$
r_{2}^{*}=\frac{b-d_{3} \theta_{1}}{s_{1} \theta_{1}\left(1-c_{1}\right)}, p_{2}^{*}=0, u_{2}^{*}=\theta_{1} \text {. }
$$

Furthermore, (I)if $\theta_{1}>\theta_{2}$, then the equilibrium $\left(r_{2}{ }^{*}, p_{2}{ }^{*}, u_{2}{ }^{*}\right)$ is unstable.

(II) If $\theta_{1}<\theta_{2}$ and

$$
\left(s_{1} r_{2}^{*}+d_{3}\right)^{2}>2 c_{1} s_{1}^{2} r_{2}^{*} \theta_{1}\left(1-\sqrt{1-c_{1}^{2}}\right)
$$

then the equilibrium $\left(r_{2}{ }^{*}, p_{2}{ }^{*}, u_{2}{ }^{*}\right) \quad$ is locally asymptotically stable for $\tau \geq 0$.

(III) If $\theta_{1}<\theta_{2}$ and

$$
\left(s_{1} r_{2}^{*}+d_{3}\right)^{2}<2 c_{1} s_{1}^{2} r_{2}^{*} \theta_{1}\left(1-\sqrt{1-c_{1}^{2}}\right)
$$

then there exists a positive number $\tau_{0}$, when $0 \leq \tau<\tau_{0}$, the equilibrium $\left(r_{2}{ }^{*}, p_{2}{ }^{*}, u_{2}{ }^{*}\right)$ is locally asymptotically stable; when $\tau>\tau_{0}$, the equilibrium $\left(r_{2}{ }^{*}, p_{2}{ }^{*}, u_{2}{ }^{*}\right)$ is unstable, and a Hopf bifurcation emerges at $\tau=\tau_{0}$.

Proof. Obviously, $r_{2}{ }^{*}$ is positive if $b / d_{3}>\theta_{1}$. Therefore, there exists a weed extinct equilibrium $\left(r_{2}{ }^{*}, p_{2}{ }^{*}, u_{2}{ }^{*}\right)$.

We consider the stability of the equilibrium $\left(r_{2}{ }^{*}, p_{2}{ }^{*}, u_{2}{ }^{*}\right)$. From (4), the characteristic equation of the linearized system is as follows 


$$
\begin{gathered}
\left(\lambda-c_{2} s_{2}\left(\theta_{1}-\theta_{2}\right)\right)\left(\lambda^{2}+\left(s_{1} r_{2}^{*}+d_{3}\right) \lambda\right. \\
\left.+c_{1} s_{1}^{2} r_{2}^{*} \theta_{1}\left(1-c_{1} e^{-\lambda \tau}\right)\right)=0 .
\end{gathered}
$$

It has one real eigenvalue $\lambda_{1}=c_{2} s_{2}\left(\theta_{1}-\theta_{2}\right)$. And its other eigenvalues are the roots of the following equation

$$
\lambda^{2}+\left(s_{1} r_{2}^{*}+d_{3}\right) \lambda+c_{1} s_{1}^{2} r_{2}^{*} \theta_{1}\left(1-c_{1} e^{-\lambda \tau}\right)=0
$$

(I) Obviously, if $\theta_{1}>\theta_{2}$, the eigenvalue $\lambda_{1}>0$. It indicates that the equilibrium $\left(r_{2}{ }^{*}, p_{2}{ }^{*}, u_{2}{ }^{*}\right)$ is unstable.

(II) If $\theta_{1}<\theta_{2}$, then the real eigenvalue $\lambda_{1}<0$. If the time delay $\tau=0$, then the two roots of (7) are

$$
\lambda_{2,3}=\frac{1}{2}\left(\begin{array}{l}
-\left(s_{1} r_{2}^{*}+d_{3}\right) \\
\pm \sqrt{\left(s_{1} r_{2}^{*}+d_{3}\right)^{2}-4 c_{1} s_{1}^{2} r_{2}^{*} \theta_{1}\left(1-c_{1}\right)}
\end{array}\right) .
$$

Obviously, the real parts of $\lambda_{2,3}$ are less than zero. Therefore, when $\tau=0$, the equilibrium $\left(r_{2}{ }^{*}, p_{2}{ }^{*}, u_{2}{ }^{*}\right)$ is locally asymptotically stable if $\theta_{1}<\theta_{2}$.

Next we consider the case $\tau>0$. Assume equation (7) has a imaginary root $\lambda=i \xi(\xi>0)$. Substituting it into (7) gives

$$
\begin{aligned}
& -\xi^{2}+i\left(s_{1} r_{2}^{*}+d_{3}\right) \xi \\
& \quad+c_{1} s_{1}^{2} r_{2}^{*} \theta_{1}\left(1-c_{1} \cos \tau \xi+i c_{1} \sin \tau \xi\right)=0
\end{aligned}
$$

Separating its real and imaginary parts yields

$$
-\xi^{2}+c_{1} s_{1}^{2} r_{2}^{*} \theta_{1}\left(1-c_{1} \cos \tau \xi\right)=0
$$

and

$$
\left(s_{1} r_{2}^{*}+d_{3}\right) \xi+c_{1}^{2} s_{1}^{2} r_{2}^{*} \theta_{1} \sin \tau \xi=0
$$

So we have

$$
\cos \tau \xi=\frac{c_{1} s_{1}^{2} r_{2}^{*} \theta_{1}-\xi^{2}}{c_{1}^{2} s_{1}^{2} r_{2}^{*} \theta_{1}}, \sin \tau \xi=-\frac{\left(s_{1} r_{2}^{*}+d_{3}\right) \xi}{c_{1}^{2} s_{1}^{2} r_{2}^{*} \theta_{1}}
$$

Based on $\sin ^{2} \tau \xi+\cos ^{2} \tau \xi=1$, one has

$$
\left(c_{1} s_{1}^{2} r_{2}^{*} \theta_{1}-\xi^{2}\right)^{2}+\left(s_{1} r_{2}^{*}+d_{3}\right)^{2} \xi^{2}=c_{1}^{4} s_{1}^{4} r_{2}^{* 2} \theta_{1}^{2}
$$

That is

$$
\begin{aligned}
\xi^{4}+\left(\left(s_{1} r_{2}^{*}+\right.\right. & \left.\left.d_{3}\right)^{2}-2 c_{1} s_{1}^{2} r_{2}^{*} \theta_{1}\right) \xi^{2} \\
& +c_{1}^{2} s_{1}^{4} r_{2}^{* 2} \theta_{1}^{2}\left(1-c_{1}^{2}\right)=0
\end{aligned}
$$

From (5), we have

$$
2 c_{1} s_{1}^{2} r_{2}^{*} \theta_{1}-\left(s_{1} r_{2}^{*}+d_{3}\right)^{2}<2 c_{1} s_{1}^{2} r_{2}^{*} \theta_{1} \sqrt{1-c_{1}^{2}} .
$$

Obviously, if $\left(s_{1} r_{2}{ }^{*}+d_{3}\right)^{2}-2 c_{1} s_{1}{ }^{2} r_{2}{ }^{*} \theta_{1} \geq 0$, then there is not any real number $\xi$ such that (9) holds. Otherwise, if $\left(s_{1} r_{2}{ }^{*}+d_{3}\right)^{2}$ $-2 c_{1} s_{1}^{2} r_{2}^{*} \theta_{1}<0$, then taking square on the two sides of (10) yields

$$
\left(2 c_{1} s_{1}^{2} r_{2}^{*} \theta_{1}-\left(s_{1} r_{2}^{*}+d_{3}\right)^{2}\right)^{2}-4 c_{1}^{2} s_{1}^{4} r_{2}^{* 2} \theta_{1}^{2}\left(1-c_{1}^{2}\right)<0
$$

So, there also is not any real number $\xi$ to make (9) hold. Therefore, the real parts of any roots of (7) must be negative for any $\tau>0$. It shows that the equilibrium $\left(r_{2}{ }^{*}, p_{2}{ }^{*}, u_{2}{ }^{*}\right)$ is locally asymptotically stable for any $\tau \geq 0$.

(III) If condition (6) holds, we have

$$
2 c_{1} s_{1}^{2} r_{2}^{*} \theta_{1}-\left(s_{1} r_{2}^{*}+d_{3}\right)^{2}>2 c_{1} s_{1}^{2} r_{2}^{*} \theta_{1} \sqrt{1-c_{1}^{2}}
$$

Taking square on the two sides yields

$$
\Delta=\left(2 c_{1} s_{1}^{2} r_{2}^{*} \theta_{1}-\left(s_{1} r_{2}^{*}+d_{3}\right)^{2}\right)^{2}-4 c_{1}^{2} s_{1}^{4} r_{2}^{* 2} \theta_{1}^{2}\left(1-c_{1}^{2}\right)>0 .
$$

Therefore, there exist two positive real number $\xi_{\text {- }}$ and $\xi_{+}$ such that (9) holds, where one might as well assume $\xi_{-} \leq \xi_{+}$. Thus,

$$
\xi_{ \pm}^{2}=\frac{1}{2}\left(2 c_{1} s_{1}^{2} r_{2}^{*} \theta_{1}-\left(s_{1} r_{2}^{*}+d_{3}\right)^{2} \pm \sqrt{\Delta}\right)
$$

From (8), it is easy to know that $\cos \tau_{-} \xi_{-}>0$. Noticed that $\sin \tau_{-} \xi_{-}<0$, so

$$
\tau_{-}=\frac{2 \pi}{\xi_{-}}-\frac{1}{\xi_{-}} \arcsin \frac{\left(s_{1} r_{2}^{*}+d_{3}\right) \xi_{-}}{c_{1}^{2} s_{1}^{2} r_{2}^{*} \theta_{1}}
$$

If $\cos \tau_{+} \xi_{+}>0$, and noticed that $\sin \tau_{+} \xi_{+}<0$, then we have

$$
\tau_{+}=\frac{2 \pi}{\xi_{+}}-\frac{1}{\xi_{+}} \arcsin \frac{\left(s_{1} r_{2}^{*}+d_{3}\right) \xi_{+}}{c_{1}^{2} s_{1}^{2} r_{2}^{*} \theta_{1}}
$$


Therefore,

$$
\tau_{0}=\min \left\{\tau_{-}, \tau_{+}\right\}=\frac{2 \pi}{\xi_{+}}-\frac{1}{\xi_{+}} \arcsin \frac{\left(s_{1} r_{2}^{*}+d_{3}\right) \xi_{+}}{c_{1}^{2} s_{1}^{2} r_{2}^{*} \theta_{1}}
$$

If $\cos \tau_{+} \xi_{+}<0$, and $\sin \tau_{+} \xi_{+}<0$, then we have

$$
\tau_{+}=\frac{\pi}{\xi_{+}}+\frac{1}{\xi_{+}} \arcsin \frac{\left(s_{1} r_{2}^{*}+d_{3}\right) \xi_{+}}{c_{1}^{2} s_{1}^{2} r_{2}^{*} \theta_{1}}
$$

Therefore,

$$
\tau_{0}=\min \left\{\tau_{-}, \tau_{+}\right\}=\frac{\pi}{\xi_{+}}+\frac{1}{\xi_{+}} \arcsin \frac{\left(s_{1} r_{2}^{*}+d_{3}\right) \xi_{+}}{c_{1}^{2} s_{1}^{2} r_{2}^{*} \theta_{1}}
$$

Next, we verify the transversal condition. Taking the derivative of $\lambda$ with respect to $\tau$ in (7), we have

$$
\frac{d \lambda}{d \tau}=-\frac{\lambda c_{1}^{2} s_{1}^{2} r_{2}^{*} \theta_{1} e^{-\lambda \tau}}{2 \lambda+s_{1} r_{2}^{*}+d_{3}+c_{1}^{2} s_{1}^{2} r_{2}^{*} \theta_{1} \tau e^{-\lambda \tau}}
$$

From (11), we obtain

$$
\begin{aligned}
\operatorname{Re}\left\{\left.\frac{d \lambda}{d \tau}\right|_{\lambda=i \xi_{+}}\right\} & =\frac{2 \xi_{+}^{2}+\left(s_{1} r_{2}^{*}+d_{3}\right)^{2}-2 c_{1} s_{1}^{2} r_{2}^{*} \theta_{1}}{\left(c_{1}^{2} s_{1}^{2} r_{2}^{*} \theta_{1}\right)^{2}} \\
& =\frac{\sqrt{\Delta}}{\left(c_{1}^{2} s_{1}^{2} r_{2}^{*} \theta_{1}\right)^{2}}>0 .
\end{aligned}
$$

Thus, the transversal condition is satisfied, hence a Hopf bifurcation occurs at $\tau=\tau_{0}$.

Case (III), $p=0$ and $u=\theta_{1}$. Similar to case (II), we have the following conclusion of the existence and stability of equilibrium.

Theorem 3. If $b / d_{3}>\theta_{2}$, then system (1) has a equilibrium $\left(r_{3}{ }^{*}, p_{3}{ }^{*}, u_{3}{ }^{*}\right)$, where

$$
r_{3}^{*}=0, p_{3}^{*}=\frac{b-d_{3} \theta_{2}}{s_{2} \theta_{2}\left(1-c_{2}\right)}, u_{3}^{*}=\theta_{2}
$$

Furthermore, (I) if $\theta_{1}<\theta_{2}$, then the equilibrium $\left(r_{3}{ }^{*}, p_{3}{ }^{*}, u_{3}{ }^{*}\right)$ is unstable.

(II) If $\theta_{1}>\theta_{2}$ and

$$
\left(s_{2} p_{3}^{*}+d_{3}\right)^{2}>2 c_{2} s_{2}^{2} p_{3}^{*} \theta_{2}\left(1-\sqrt{1-c_{2}^{2}}\right)
$$

then the equilibrium $\left(r_{3}{ }^{*}, p_{3}{ }^{*}, u_{3}{ }^{*}\right)$ is locally asymptotically stable for $\tau \geq 0$.

(III) If $\theta_{1}>\theta_{2}$ and

$$
\left(s_{2} p_{3}^{*}+d_{3}\right)^{2}<2 c_{2} s_{2}^{2} p_{3}^{*} \theta_{2}\left(1-\sqrt{1-c_{2}^{2}}\right)
$$

then there exist a positive number $\tau_{0}$, when $0 \leq \tau<\tau_{0}$, the equilibrium $\left(r_{3}{ }^{*}, p_{3}{ }^{*}, u_{3}{ }^{*}\right)$ is locally asymptotically stable; when $\tau>\tau_{0}$, the equilibrium $\left(r_{3}{ }^{*}, p_{3}{ }^{*}, u_{3}{ }^{*}\right)$ is unstable, and a Hopf bifurcation emerges at $\tau=\tau_{0}$.

\section{EXAMPLES}

According to those discussion in Section III, we give two examples to illustrate the correctness of our results.

Example 1. In system (1), let $c_{1}=0.8, c_{2}=0.3, s_{1}=6, s_{2}=2$, $b=5, d_{1}=0.2, d_{2}=0.7, d_{3}=0.2$ and $\tau=5$. Then system (1) has three equilibria as follows, the paddy and weed extinct equilibrium $(0,0,25)$, the paddy extinct equilibrium $(0,2.9184$, $1.1667)$, and the weed extinct equilibrium $((99.8333,0,0.0417)$.

By computing, we have $b / d_{3}=25, \theta_{1} \approx 0.0417$ and $\theta_{2} \approx 1.1667$. So the inequality $b / d_{3}>\theta_{2}>\theta_{1}$ holds. We can verify inequalitie (5) hold. From Theorem 1-3, the equilibria $(0,0,25)$ and $(0,2.9184,1.1667)$ are unstable, and the weed extinction equilibrium $(99.8333,0,0.0417)$ is asymptotically stable for any $\tau \geq 0$.

Example 2. In system (1), let $c_{1}=0.5, c_{2}=0.3, s_{1}=0.6, s_{2}=0.01$, $b=0.015, d_{1}=0.2, d_{2}=0.99, d_{3}=0.01$ and $\tau=5$. By computing, we have $b / d_{3}=1.5, \theta_{1} \approx 0.6667$ and $\theta_{2} \approx 330$. So the inequality $\theta_{2}>$ $b / d_{3}>\theta_{1}$ holds. Then system (1) has two equilibria as follows, the paddy and weed extinct equilibrium $(0,0,1.5)$, and the weed extinct equilibrium $(0.0417,0,0.6667)$.

It is not difficult to verify inequality (6) holds. From (13), we obtain $\tau_{0} \approx 65.4899$. Therefore, by Theorem 2 , the equilibria $(0.0417,0,0.6667)$ is asymptotically stable when $0 \leq \tau<\tau_{0}$ (see Fig. 1(A)); when $\tau>\tau_{0}$, the equilibrium $\quad(0.0417,0$, 0.6667) is unstable, and a Hopf bifurcation emerges at $\tau=\tau_{0}$. (see Fig. 1(B)). 

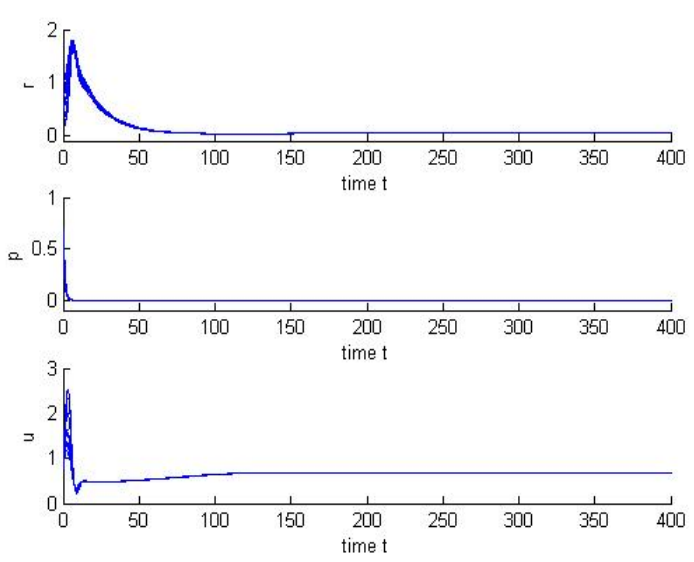

(A)
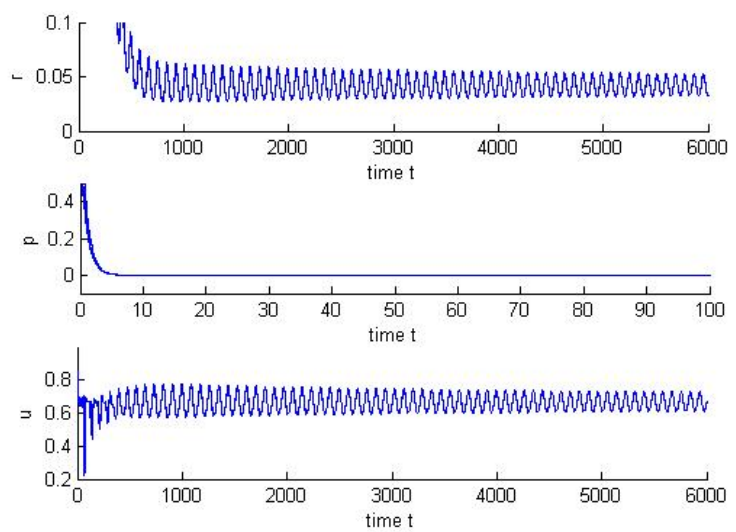

(B)

FIGURE I. TIME RESPONSE CURVES OF EXAMPLE $2, \mathrm{C} 1=0.5, \mathrm{C} 2=0.3$ $\mathrm{S} 1=0.6, \mathrm{~S} 2=0.01, \mathrm{~B}=0.015, \mathrm{D} 1=0.2, \mathrm{D} 2=0.99$ AND D3 $=0.01$. (A) $\mathrm{T}=5$, (B) $\mathrm{T}=65.5$

\section{CONCLUSIONS}

We have proposed a differential equation model that reflects the interaction among rice, weed and inorganic fertilizer in the paddy ecosystem. In this system, a rice and weed extinct equilibrium $\left(0,0, u_{1}{ }^{*}\right)$ always exists, and the equilibrium is stable under condition $b / d_{3}<\min \left\{\theta_{1}, \theta_{2}\right\}$. Otherwise, it is unstable. At this time, if $\theta_{1}>\theta_{2}$, then when $b / d_{3}>\theta_{2}$, system (1) has a rice extinction equilibrium $\left(0, p_{2}{ }^{*}\right.$, $\left.u_{2}{ }^{*}\right)$, which is asymptotically stable; when $b / d_{3}>\theta_{1}$, system (1) still has a weed extinction equilibrium $\left(r_{3}{ }^{*}, 0, u_{3}{ }^{*}\right)$, which is unstable. If $\theta_{1}<\theta_{2}$, then when $b / d_{3}>\theta_{1}$, system (1) has a weed extinction equilibrium $\left(r_{3}{ }^{*}, 0, u_{3}{ }^{*}\right)$, which is asymptotically stable, when $b / d_{3}>\theta_{2}$, system (1) still has a rice extinction equilibrium $\left(0, p_{2}{ }^{*}, u_{2}{ }^{*}\right)$, which is unstable. Our results show that the existence and stability of equilibrium points are related to the relative mortality of rice and weed, $\theta_{1}$ and $\theta_{2}$, and to the ratio of fertilizer supply and loss $b / d_{3}$. The presence of time delay $\tau$ maybe drive the system to instability.

In the paddy field management, we should take some measures to make the weed extinction equilibrium $\left(r_{3}{ }^{*}, 0, u_{3}{ }^{*}\right)$, exist and be stable. Obviously, at this time the equilibria $(0,0$, $\left.u_{1}{ }^{*}\right)$ and $\left(0, p_{2}{ }^{*}, u_{2}{ }^{*}\right)$ are not stable or not exist. According to
Theorem 3 , these measures include: reducing the loss rate $d_{3}$ of inorganic fertilizer, increasing fertilization rate $b$, selecting rice varieties with low mortality $d_{1}$, increasing mortality of weed $\mathrm{d} 2$, reducing $t$ the utilization rate of light energy of weed. We also can reduce the rice mortality rate $d_{1}$, increase the utilization rate of light energy of rice $c_{1}$. From the expression of $r_{3}{ }^{*}$, these measures also help to increase rice yield.

\section{ACKNOWLEDGMENT}

This work was supported in part by Hunan province science and technology project (grants 2015JC3101) and Hunan province graduate student innovation training project (grants CX2015B265).

\section{REFERENCES}

[1] P. Su, X. Liao, Y. Zhang, and H. Huang, "Influencing factors on rice sheath blight epidemics in integrated rice-duck system," J. Integ. Agri., vol. 11, pp. 1462-1473, Sep.2012.

[2] Q.Teng, X.F. Hu, C.Cheng, et al. "Ecological effects of rice-duck integrated farming on soil fertility and weed and pest control," J. Soils Sedi., vol. 16, pp.2395-2407, Oct. 2016.

[3] Z. Yang, X. Liu, H. Huang, et al. "A study on the influence of rice-duck intergrowth on spider, rice diseases, insect and weeds in rice-duck complex ecosystem," Acta Ecol. Sinica, vol. 24, pp.2756-2760, Dec.2004. (in Chinese).

[4] C. Dai, M. Zhao, and H. Yu, "Dynamics induced by delay in a nutrientphytoplankton model with diffusion," Ecol. Complexity, vol.26, pp.2936, Jun.2016.

[5] H. Zhao, X. Huang, and X. Zhang, "Hopf bifurcation and harvesting control of a bioeconomic plankton model with delay and diffusion terms," Physica A: Stat. Mech. Appl., vol. 421, pp.300-315, Mar. 2015.

[6] J. Zhao, J. P. Tian, and J. Wei, "Minimal model of plankton systems revisited with spatial diffusion and maturation delay, " Bull. Math. Biol., vol. 78, pp. 381-412, Mar.2016.

[7] Y. Sekerci, S. Petrovskii, "Mathematical modelling of planktoncoxygen dynamics under the climate change," Bull. Math. Biol., vol. 77, pp. 2325-2353, Dec.2015. 